\title{
Some Results on Invariant Submanifolds in an Indefinite Trans-Sasakian Manifold-II
}

\author{
Nanditha S Matad \\ New Horizon College, Marathalli, Bangalore-560103, Karnataka, INDIA
}

\begin{abstract}
The purpose of this paper is to study invariant submanifolds in a indefinite trans-Sasakian manifold. Necessary and sufficient conditions are given on an submanifold of a indefinite trans-Sasakian manifold to be invariant and invariant case is considered. In this case further properties and some theorems are given related to an invariant submanifolds in a indefinite transSasakian manifold.
\end{abstract}

AMS Subject Classification : 53C15, 53C20, $53 C 25$.

Keywords: Indefinite Trans-Sasakian manifold, Invariant submanifold, Covariant differentiation

\section{Introduction}

In general, the geometry an invariant submanifold inherits almost all properties of the ambient manifold. In 1976 K.Yano and M.Kon introduced invariant and anti-invariant sub- manifolds in [1]. J.A.Oubina [2] introduced the notion of a tran sasakian manifold of type $(\alpha, \beta)$. Trans sasakian manifold is an important kind of sasakian manifold such that $\alpha=1$ and $\beta=1$. In 1998, Xu.Xufeng and Chao Xiadi proved that every - sasakian manifold is a hyper surfaces of an indefinite Khalerian manifold and established a necessary and sufficient condition for an odd dimensional Riemannian manifold to be an - sasakian manifold [3]. In [4]U.C.De and Avijit Sarkar introduced and studied the notion of Kenmotsu manifolds with indefinite metric with an example.

In 2010 S.S.Shukla and D.D.Singh [5]Studies -Trans sasakian manifolds. In this paper they have obtained some results on -Trans sasakian manifolds.Conditions for Indefinite trans sasakian manifolds to be D-totally geodesics, $\mathrm{D} \perp$-totally geodesics, mixed totally geodesic is given by Arindam Bhattacharya and Bandana Das in [7].

Recently some basic results on locally $\varphi$-recurrent,locally $\varphi$ symmetric and $\varphi$ quasi confor- maly symmetric Indefinite tran sasakian manifolds have been obtained by Venkatesha and sumangala.B.[6]. In 2015 Shyam kishor and Abhishek Singh [8]has studied on weekly con- circular symmetric and weakly concircular Ricci symmetric 3-dimensional - tran sasakian manifold. Avijit Sarkara and Matilal Sen[10] has obtained invariant submanifolds with the second fundamental form satisfying some conditions and difference between the conditions for submanifolds of $\alpha$-Sasakian and
$\beta$-Kenmotsu manifolds to be totally geodesic is shown. In this paper necessary and sufficient conditions are given on an submanifold of an indefinite trans-Sasakian manifold to be invariant and further properties and some theorems are given related to an invariant submanifolds in a indefinite trans-Sasakian manifold.

\section{Preliminaries}

Let $M$ be an $(2 n+1)$-dimensional indefinite almost contact metric manifold with indefinite almost contact metric structure $(\varphi, \xi, \eta, g)$ then they satisfies

$$
\begin{gathered}
\varphi^{2}=-\mathrm{I}+\eta \otimes \xi \\
\eta(\xi)=1, \varphi \xi=0 \\
\mathrm{~g}(\varphi \mathrm{X}, \varphi \mathrm{Y})=\mathrm{g}(\mathrm{X}, \mathrm{Y})-\varepsilon \eta(\mathrm{X}) \eta(\mathrm{Y}), \\
\mathrm{g}(\varphi \mathrm{X}, \mathrm{Y})=-\mathrm{g}(\mathrm{X}, \varphi \mathrm{Y}) \\
\varepsilon \mathrm{g}(\mathrm{X}, \xi)=\eta(\mathrm{X})
\end{gathered}
$$

Where $\mathrm{X}, \mathrm{Y}$ are vector fields on $\mathrm{M}$ and $\varepsilon= \pm 1$

An indefinite almost contact metric structure $(\varphi, \xi, \eta, g)$ on $M$ is called indefinite trans- Sasakian if

$(\nabla \mathrm{X} \varphi)(\mathrm{Y})=\alpha[\mathrm{g}(\mathrm{X}, \mathrm{Y}) \xi-\varepsilon \eta(\mathrm{Y}) \mathrm{X}]+\beta[\mathrm{g}(\varphi \mathrm{X}, \mathrm{Y}) \xi-\varepsilon \eta(\mathrm{Y}) \varphi \mathrm{X}]$

Where $\alpha$ and $\beta$ are non zero scalar functions on $\bar{M}$ of type $(\alpha, \beta v)$ is a Levi-civita connection on $\bar{M}$. In particular, an indefinite trans-Sasakian manifold is normal.

From above formula, one easily obtains

$$
\begin{aligned}
& \nabla_{\mathrm{X}} \xi=-\alpha \varepsilon \varphi \mathrm{X}+\beta \mathrm{X}-\varepsilon \eta(\mathrm{X}) \xi=\varepsilon\left[-\alpha \varphi \mathrm{X}+\varphi^{2} \mathrm{X}\right], \\
& \nabla_{\mathrm{X}} \eta(\mathrm{Y})=-\alpha \mathrm{g}(\varphi \mathrm{X}, \mathrm{Y})+\beta[\mathrm{g}(\mathrm{X}, \mathrm{Y})-\varepsilon \eta(\mathrm{X}) \eta(\mathrm{Y})]
\end{aligned}
$$

Further in an indefinite trans sasakian manifold, the following holds true,

$$
\mathrm{R}(\mathrm{X}, \mathrm{Y}) \dot{\xi}=\left(\alpha^{2}-\beta^{2}\right)[\eta(\mathrm{Y}) \mathrm{X}-\eta(\mathrm{X}) \mathrm{Y}]+2 \alpha \beta[\eta(\mathrm{Y}) \varphi \mathrm{X}-\eta(\mathrm{X}) \varphi \mathrm{Y}
$$

Let $\mathrm{M}$ be an $(2 \mathrm{~m}+1)$ dimensional $(\mathrm{n}>\mathrm{m})$ manifold imbedded in $\mathrm{M}$. The induced metric $\mathrm{g}$ of $\mathrm{M}$ is given $\mathrm{g}(\mathrm{X}$, $\mathrm{Y})=\mathrm{g}(\mathrm{X}, \mathrm{Y})$ for any vector fields $\mathrm{X}, \mathrm{Y}$ on $\mathrm{M}$.
Let $\mathrm{T}_{\mathbf{X}}(\mathrm{M})$ and $\mathrm{T}_{\mathbf{X}}(\mathrm{M})^{\perp}$ denote that tangent and normal bundles of $M$ and $x \in M$. Let $\nabla X$ denote the Riemannian connection on $\mathrm{M}$ determined by the induced metric $\mathrm{g}$ and $\mathrm{R}$ denote the Riemannian curvature tensor of $\mathrm{M}$. Then Gauss-Weingarten formula is given by 


\section{International Journal of Science and Research (IJSR) \\ ISSN (Online): 2319-7064}

Index Copernicus Value (2016): 79.57 | Impact Factor (2015): 6.391

$$
\begin{aligned}
& \bar{\nabla}_{\mathrm{X}} \mathrm{Y}=\nabla_{\mathrm{X}} \mathrm{Y}+\mathrm{B}(\mathrm{X}, \mathrm{Y}), \\
& \bar{\nabla}_{\mathrm{X}} \mathrm{N}=-\mathrm{A}_{\mathrm{N}}(\mathrm{X})+\mathrm{D}_{\mathrm{X}} \mathrm{N}
\end{aligned}
$$

for any vector fields $\mathrm{X}, \mathrm{Y}$ tangent to $\mathrm{M}$ and any vector field $\mathrm{N}$ normal to $\mathrm{M}$, where $\mathrm{D}$ is the operator of covariant differentiation with respect to the linear connection induced in the normal bundle $\mathrm{T}_{\mathrm{X}}(\mathrm{M})^{\perp}$. Both $\mathrm{A}$ and $\mathrm{B}$ are called the second fundamental forms of they satisfy

$$
\mathrm{g}(\mathrm{B}(\mathrm{X}, \mathrm{Y}), \mathrm{N})=\mathrm{g}\left(\mathrm{A}_{\mathrm{N}}(\mathrm{X}, \mathrm{Y})\right) \text {. }
$$

\section{Invariant Submanifolds of an indefinite trans-Sasakian manifold}

Let $\mathrm{M}$ be an immersed submanifold of an indefinite transSasakian manifold $\bar{M}$. If $\varphi\left(B\left(T_{X} M\right)\right) \subset T_{X} M$, for any point $\mathrm{x} \in \mathrm{M}$, then $\mathrm{M}$ is called an invariant submanifold of $\overline{\mathrm{M}}$.

In this case, we have

$$
\begin{aligned}
\varphi \mathrm{BX} & =\underset{t}{\mathrm{~B}} \phi \mathrm{X}, \\
\varphi \mathrm{N}_{1} & =\sum_{l=1}^{t} \lambda_{15} \mathrm{~N}_{5}, \\
\xi & =\mathrm{BV}+\Sigma_{\alpha_{1}}^{t} \mathrm{~N}_{5} .
\end{aligned}
$$$$
1=1
$$

Let $\nabla$ be the Levi-civita connection of $\mathrm{M}$ with respect to the induced metric g. Then theGauss and Weingarten formulas are given by

$$
\begin{aligned}
& \bar{\nabla}_{\mathrm{X}} \xi=\nabla_{\mathrm{X}} \xi+\mathrm{h}(\mathrm{X}, \mathrm{Y}), \\
& \nabla_{\mathrm{X}} \mathrm{N}=\nabla^{\perp} \mathrm{N}-\mathrm{A}_{\mathrm{N}} \mathrm{X} .
\end{aligned}
$$

for any $\mathrm{X}, \mathrm{Y} \in \Gamma(\mathrm{T} \mathrm{M})$ and $\mathrm{N} \in \Gamma(\mathrm{T} \mathrm{M})^{\perp} \cdot \nabla^{\perp}$ is the connection in the normal bundle, $h$ is the second fundamental form of $\mathrm{M}$ and $\mathrm{A}_{\mathrm{N}}$ is the weigarten endomorhism associated with $\mathrm{N}$.The second fundamental form $\mathrm{h}$ and the shape operator $\mathrm{A}$ related by,

$$
\mathrm{g}(\mathrm{h}(\mathrm{X}, \mathrm{Y}), \mathrm{N})=\mathrm{g}\left(\mathrm{A}_{\mathrm{N}} \mathrm{X}, \mathrm{Y}\right) \text {. }
$$

Lemma3.1. Let $M$ be an invariant submanifold of a indefinite trans sasakian manifold $\overline{\mathrm{M}}$, then we have,

for any $\mathrm{X} \in \mathrm{T} \overline{\mathrm{M}}$.

$$
\mathrm{h}(\mathrm{X}, \xi)=0
$$

Proof: For any indefinite trans sasakian manifold using (3.4)we get

$$
\nabla_{\mathrm{X}} \xi=\varepsilon[-\alpha \varphi \mathrm{X}+\beta(\mathrm{X}-\eta(\mathrm{X}) \xi)]
$$

on the other hand, we get

$$
\nabla_{\mathrm{X}} \xi+\mathrm{h}(\mathrm{X}, \xi)=\varepsilon[-\alpha \varphi \mathrm{X}+\beta(\mathrm{X}-\eta(\mathrm{X}) \xi)]=
$$

from the gauss formula then the equation is implied that $\mathrm{h}(\mathrm{X}, \xi)=0$.

Theorem 3.1. Let $M$ be an invariant submanifolds of an indefinite trans sasakian manifold $\overline{\mathrm{M}}$ then we have

$$
\mathrm{l}(\varphi \mathrm{X}, \mathrm{Y})=\bar{\varphi}(\mathrm{h}(\mathrm{X}, \mathrm{Y}))=\mathrm{h}(\mathrm{X}, \varphi \mathrm{Y}) \text {. }
$$

for any $X, Y \in \Gamma(T M$.)

$$
\begin{aligned}
& \left(\nabla_{\mathrm{X}} \varphi\right) \mathrm{Y}=\nabla_{\mathrm{X}} \varphi \mathrm{Y}-\varphi\left(\overline{\mathrm{V}}_{\mathrm{X}} \mathrm{Y}\right), \\
& =\nabla_{\mathrm{X}} \varphi \mathrm{Y}+\mathrm{B}(\mathrm{X}, \varphi \mathrm{Y})-\varphi\left(\nabla_{\mathrm{X}}, \mathrm{Y}\right)-\varphi(\mathrm{B}(\mathrm{X}, \mathrm{Y})),
\end{aligned}
$$$$
=\left(\nabla_{X} \varphi\right) Y+B(X, \varphi \mathrm{Y})-\varphi(B(X, Y)) . \quad \text { Issue 12, December } 2017
$$

thus we get
Then we have

$\alpha(\mathrm{g}(\mathrm{X}, \mathrm{Y}) \xi-\varepsilon \eta(\mathrm{Y}) \mathrm{X})+\beta(\mathrm{g}(\varphi \mathrm{X}, \mathrm{Y}) \xi-\varepsilon \eta(\mathrm{Y}) \varphi \mathrm{X})$

$=\alpha(\mathrm{g}(\mathrm{X}, \mathrm{Y}) \xi-\varepsilon \eta(\mathrm{Y}) \mathrm{X})+\beta(\mathrm{g}(\varphi \mathrm{X}, \mathrm{Y}) \xi-\varepsilon$

$\eta(\mathrm{Y}) \varphi \mathrm{X})+\mathrm{B}(\mathrm{X}, \varphi \mathrm{Y})-\varphi(\mathrm{B}(\mathrm{X}, \mathrm{Y}))$

$\mathrm{B}(\mathrm{X}, \varphi \mathrm{Y})=\varphi(\mathrm{B}(\mathrm{X}, \mathrm{Y}))$

on the other hand, it follows that

$\left(\nabla_{\mathrm{Y}} \varphi\right) \mathrm{X}=\left(\nabla_{\mathrm{Y}} \varphi\right) \mathrm{X}+\mathrm{B}(\mathrm{Y}, \varphi \mathrm{X})-\varphi(\mathrm{B}(\mathrm{Y}, \mathrm{X}))$

Hence we have $\mathrm{h}(\varphi \mathrm{X}, \mathrm{Y})=\bar{\varphi}(\mathrm{h}(\mathrm{X}, \mathrm{Y}))=\mathrm{h}(\mathrm{X}, \varphi \mathrm{Y})$

Lemma 3.2. Let $M$ be an invariant submanifolds of an indefinite trans sasakian manifold $\bar{M}$ then we have

$$
\mathrm{h}(\varphi \mathrm{X}, \varphi \mathrm{Y})=-\mathrm{h}(\mathrm{X}, \mathrm{Y})
$$

Proof: From (3.8) and (2.1) we get

$$
\begin{aligned}
\mathrm{h}(\varphi \mathrm{X}, \emptyset \mathrm{Y}) & =\varphi(\mathrm{h}(\mathrm{X}, \emptyset \mathrm{Y}))=\varphi^{2}(\mathrm{~h}(\mathrm{X}, \mathrm{Y})), \\
& =-\mathrm{h}(\mathrm{X}, \mathrm{Y})+\eta(\mathrm{h}(\mathrm{X}, \mathrm{Y})) \xi=-\mathrm{h}(\mathrm{X}, \mathrm{Y})
\end{aligned}
$$

Lemma 3.3. If $X$ is an arbitary tangent vector of an invariant submanifold of an indefinite trans sasakian,then $\nabla_{X} \xi$ is also an arbitrary tangent vector different from a vector spanned by $\xi$.

Proof: from 2.5 we get,

$$
\nabla \mathrm{X} \xi=\beta \varepsilon \mathrm{X}-\alpha \varepsilon \varphi \mathrm{X}-\beta \varepsilon \eta(\mathrm{X}) ;
$$

From the above equation we see that $\nabla \mathrm{X} \xi$ is a linear combination of $\mathrm{X}, \varphi \mathrm{X}$, and $\xi$. Since an invariant submanifold of an indefinite trans sasakian manifold is also an indefinite trans sasakian manifold, the dimention of the submanifold is odd. Hence we can consider that an orthonormal $\varphi$-basis[9]. $\left[\mathrm{e}_{\mathbf{i}}, \varphi \mathrm{e}_{\mathbf{i}}, \xi\right], \mathrm{i}=1,2,3, \ldots . . \mathrm{n}$ is the dimension of the submanifold, and we can write

$$
\begin{gathered}
X=\sum_{i=1} a_{i} b_{i}+\sum b_{i} \varphi e_{i}+c \xi . \\
\varphi X=-\Sigma b_{i} e_{i}+\Sigma a_{i=1} \varphi e_{i} .
\end{gathered}
$$

Here $a_{\mathbf{i}}, b_{\mathbf{i}}$ and $c$ are scalars. From (3.10), (3.11) and (3.12) we obtain

$$
\left.\nabla_{\mathrm{X}} \xi=\varepsilon \underset{\mathrm{i}=1}{\varepsilon[\Sigma}\left(\beta \mathrm{a}_{\mathrm{i}}+\alpha \mathrm{b}_{\mathrm{i}}\right) \mathrm{e}_{\mathrm{i}}+\Sigma\left(\beta \mathrm{b}_{\mathrm{i}=1}-\alpha \mathrm{a}_{\mathrm{i}}\right) \varphi \mathrm{e}_{\mathrm{i}}\right]
$$

If we write $\mathrm{TM}=\mathrm{D} \oplus\langle\xi\rangle$, we see that $\nabla \mathrm{X} \xi \in \mathrm{D}$ and, if $\mathrm{X}$ is arbitrary, $\nabla \mathrm{X} \xi$ is also arbitrary, because $\mathrm{a}_{\mathbf{i}}, \mathrm{b}_{\mathbf{i}}$ and $\mathrm{c}$ are arbitrary.

If we write $\mathrm{TM}=\mathrm{D} \oplus\langle\xi\rangle$, we see that $\nabla \mathrm{X} \xi \in \mathrm{D}$ and, if $\mathrm{X}$ is arbitrary, $\nabla_{\mathrm{X}} \xi$ is also arbitrary, because $\mathrm{a}_{\mathbf{i}}, \mathrm{b}_{\mathbf{i}}$ and $\mathrm{c}$ are arbitrary.

Theorem 3.2: Let $M$ be an immersed submanifolds of an indefinite trans sasakian manifold $\bar{M}$ then we have the second fundamental form $h$ is $\eta$-parallel if 


\section{International Journal of Science and Research (IJSR)}

ISSN (Online): 2319-7064

Index Copernicus Value (2016): 79.57 | Impact Factor (2015): 6.391

$(\nabla \mathrm{X} h)(\mathrm{Y}, \mathrm{Z})=\alpha[\varepsilon \eta(\mathrm{Y}) \varphi \mathrm{h}(\mathrm{X}, \mathrm{Z})+\varepsilon \eta(\mathrm{Z}) \varphi \mathrm{h}(\mathrm{X}, \mathrm{Y})]-\beta[\varepsilon \eta(\mathrm{Y}) \mathrm{h}(\mathrm{X}, \mathrm{Z})+\varepsilon \eta(\mathrm{Z}) \mathrm{h}(\mathrm{X}, \mathrm{Y})]$

Proof: We know that

$\left(\nabla_{\mathrm{X}} \mathrm{h}\right)(\varphi \mathrm{Y}, \varphi \mathrm{Z})=\left(\nabla_{\mathrm{X}} \mathrm{h}\right)(\varphi \mathrm{Y}, \varphi \mathrm{Z})-\mathrm{h}\left(\nabla_{\mathrm{X}} \varphi \mathrm{Y}, \varphi \mathrm{Z}\right)-\mathrm{h}\left(\varphi \mathrm{Y}, \nabla_{\mathrm{X}} \varphi \mathrm{Z}\right)$,

Then

$\left(\nabla_{\mathrm{X}} \mathrm{h}\right)(\varphi \mathrm{Y}, \varphi \mathrm{Z})=\mathrm{h}\left(\nabla_{\mathrm{X}} \varphi \mathrm{Y}, \varphi \mathrm{Z}\right)+\mathrm{h}\left(\varphi \mathrm{Y}, \nabla_{\mathrm{X}} \varphi \mathrm{Z}\right)$

Using (3.9)

$-\left(\nabla_{X} h\right)(Y, Z)=h\left[\left(\nabla_{X} \varphi\right) Y+\varphi\left(\nabla_{X} Y\right), \varphi Z\right]+h\left[\varphi \mathrm{Y},\left(\nabla_{X} \varphi\right) Z+\varphi\left(\nabla_{X} Z\right)\right]$,

$-\left(\nabla_{X} h\right)(Y, Z)=h\left(\left(\nabla_{X} \varphi\right) Y, \varphi Z\right)-h\left(\nabla_{X} Y, Z\right)+h\left(\varphi Y,\left(\nabla_{X} \varphi\right) Z\right)-h\left(Y, \nabla_{X} Z\right)$,

Thus by using (2.4)we have

$-\left(\nabla_{\mathrm{x}} \mathrm{h}\right)(\mathrm{Y}, \mathrm{Z})=-\alpha \varepsilon \eta(\mathrm{Y}) \mathrm{h}(\mathrm{X}, \varphi \mathrm{Z})-\beta \varepsilon \eta(\mathrm{Y}) \mathrm{h}(\varphi \mathrm{X}, \varphi \mathrm{Z})-\alpha \varepsilon \eta(\mathrm{Z}) \mathrm{h}(\varphi \mathrm{Y}, \mathrm{X})-\beta \varepsilon \eta(\mathrm{Z}) \mathrm{h}(\varphi \mathrm{Y}, \varphi \mathrm{X})$,
$\left(\nabla_{\mathrm{x}} \mathrm{h}\right)(\mathrm{Y}, \mathrm{Z})=\alpha[\varepsilon \eta(\mathrm{Y}) \varphi \mathrm{h}(\mathrm{X}, \mathrm{Z})+\varepsilon \eta(\mathrm{Z}) \varphi \mathrm{h}(\mathrm{X}, \mathrm{Y})]-\beta[\varepsilon \eta(\mathrm{Y}) \mathrm{h}(\mathrm{X}, \mathrm{Z})+\varepsilon \eta(\mathrm{Z}) \mathrm{h}(\mathrm{X}, \mathrm{Y})]$

Hence the proof.

\section{References}

[1] K.Yano and M.Kon, Anti invariant submanifolds Marcel Dekker Inc., New York,(1976).

[2] J.A.Oubina, New classes of almost contact metric structures, Publ.Math.Debrecen.,32,(1985),187-193.

[3] X.Xufeng and C.Xiaoli, Two theorems on sasakian manifolds, Int.J.Math.Sci., 21(2) (1998), 249-254.

[4] U.C.De and Avijit Sarkar, On Kenmotsu manifolds, Hadronic Journal., 32(2009), 231-242.

[5] S.S.Shukla and D.D.Singh , On -Trans sasakian manifolds, Int.Journal.,32,(2009), 231-242.

[6] Venkatesha and Sumangala.B. , Some results on Trans Sasakian manifolds, International Journal of Mathematics and Statistics invention., Volume 2, issue 5,(2014), 22-26.

[7] ArindamBhattacharya and Bandana Das , Contact CR-Submanifolds of an Indefinite trans sasakian manifolds,

Int.J.Contemp.Math.Science., Volume.6,no.26,(2011), 1271-1282.

[8] Shyam kishor and Abhishek Singh, On weekly concircular symmetric and weakly concircular Ricci sym- metric 3-dimensional - tran sasakian manifold, IJMAA., Volume 3, issue 4,(2015), 65-73.

[9] Blair.D.E., Riemannian Geometry of Contact and Symplectic Manifold, Boston,Berlin,(2002).

[10] Avijith Sarkar and Matilal , On invariant submanifolds of Trans sasakian manifolds, Proceedings of the

[11]Estonian Academy of Sciences,61,1,(2012),29-37.

Volume 6 Issue 12, December 2017

www.ijsr.net

Licensed Under Creative Commons Attribution CC BY 